\title{
Acciones educativas desde las comunidades de aprendizaje, un proceso formativo dialógico y participativo de maestros
}

Educational actions from the learning communities, a dialogic and participatory training process for teachers

Ações educativas junto às comunidades de aprendizagem, processo formativo dialógico e participativo de professores

ARTÍCULO DE REVISIÓN

\author{
Pepe Delgadillo Balderrama \\ pepitodelgadillob@gmail.com \\ ORCID: 0002-6022-903X
}

Universidad Autónoma del Beni “José Ballivián”, Bolivia

Recibido 03 de diciembre 2020 | Arbitrado y aceptado 15 de diciembre 2020 | Publicado en diciembre 2020

\section{RESUMEN}

En las Escuelas Superiores en Bolivia, es importante priorizar acciones educativas desde las comunidades de aprendizaje como un proceso formativo dialógico y participativo de maestros. El estudio tuvo como principal objetivo determinar el tipo de atención que brindan los docentes a los estudiantes en estas instituciones con relación a la formación pedagógica y didáctica. El método aplicado fue dialecticomaterialista, sobre la base del resto de los métodos teóricos y empíricos. Como desarrollo y análisis de los resultados se obtuvo que el 84\% de los actores educativos manifiestan que la formación de maestros es aislada e individualista, no existe colaboración, ni diálogo participativo en el accionar formativo de maestros, por lo que los entrevistados y encuestados se ratifican en esta postura. Se concluye determinando que la acciones educativas posibilitan desarrollar la formación de maestros en la interacción dialógica y participativa de experiencias.

Palabras clave: Acciones educativas; escuelas superiores; comunidades; dialógico, participativo

\section{ABSTRACT}

In Higher Schools in Bolivia, it is important to prioritize educational actions from the learning communities as a dialogic and participatory training process for teachers. The main objective of the study was to determine the type of attention that teachers provide to students in these institutions in relation to pedagogical and didactic training. The applied method was dialectical-materialistic, based on the rest of the theoretical and empirical methods. As development and analysis of the results, it was obtained that $84 \%$ of the educational actors state that teacher training is isolated and individualistic, there is no collaboration, or participatory dialogue in the training of teachers, and so the interviewees and respondents are they ratify this position. It is concluded by determining that educational actions make it possible to develop teacher training in the dialogic and participatory interaction of experiences.

Key words: Educational actions; higher schools; communities; dialogical, participatory 


\section{RESUMO}

Nas Escolas Superiores da Bolívia, é importante priorizar as ações educativas das comunidades de aprendizagem como um processo dialógico e participativo de formação de professores. 0 objetivo principal do estudo foi determinar o tipo de atenção que os professores dispensam aos alunos dessas instituições em relação à formação pedagógica e didática. 0 método aplicado foi dialético-materialista, com base nos demais métodos teóricos e empíricos. Quanto ao desenvolvimento e análise dos resultados, obteve-se que $84 \%$ dos atores educacionais afirmam que a formação de professores é isolada e individualista, não há colaboração, nem diálogo participativo na formação de professores, portanto os entrevistados e respondentes são eles ratificam esta posição. Conclui-se determinando que as ações educativas possibilitam o desenvolvimento da formação de professores na interação dialógica e participativa das experiências.

Palavras-chave: Ações educativas; escolas superiores; comunidades; dialógico, participativo

\section{INTRODUCCIÓN}

Las instituciones de educación superior formadoras de maestros son, sin duda, los centros que se encargan de realizar diversas actividades formativas encaminadas a lograr habilidades pedagógicas y didácticas, a la par del avance de las tecnologías de la comunicación para lograr los propósitos institucionales sin desconocer la trascendencia de la función educativa. Este es un proceso de vital importancia, ya que permite el mejoramiento de la calidad de los servicios educativos que puede ofrecer el futuro maestro al mercado laboral en lo que respecta la educación a favor del crecimiento humano en la sociedad como proceso y resultado en toda su complejidad.

Por lo que, desde siempre la formación de maestros en instituciones de Educación Superior, desarrolla fundamentalmente lo que es la labor del futuro maestro, para que los estudiantes en educación escolar aprendan y trasciendan el conocimiento desde el desarrollo educativo inclusivo, diverso y de calidad, utilizando estrategias educativas en aula, compartiendo experiencias pedagógicas $\mathrm{y}$ didácticas entre colegas maestros que es lo ideal.

Por lo tanto, las Escuelas Superiores de Formación de maestros tienen como misión esencial la formación de profesionales educadores altamente capacitados que desempeñen sus roles como ciudadanos responsables, competentes y comprometidos con el desarrollo social y cultural, ello implica que en el proceso de formación de los maestros de cualquier nivel educativo, estén preparados en las diversas habilidades educativas que permita cumplir con las funciones que requiere su profesión como educador.

El objetivo principal del estudio determinar el tipo de atención que brindan los docentes a los estudiantes futuros maestros en los aspectos pedagógicos y didácticos en las instituciones de educación superior.

En este contexto, la investigación caracteriza el proceso formativo de maestros y las dificultades que atraviesa, y como producto de análisis y reflexión de la problemática constatada que consiste en lo aislado e individualista, nada colaborativo, ni dialógicoparticipativo de la educación en las escuelas superiores en Bolivia. se considera implementar acciones educativas desde las comunidades de aprendizaje, lo cual contribuye a desarrollar un proceso formativo interactivo, con la finalidad de compartir experiencias educativas innovadoras en lo pedagógico y didáctico en las Escuelas Superiores, generando una educación con sentido y calidad en el marco del diálogo, la participación y el trabajo colaborativo de cooperación interinstitucional.

MÉTODO

La investigación se sustenta en lo dialectico - materialista a partir de los métodos teóricos enmarcados en lo (histórico - lógico, inductivo - 
deductivo, sistémico - estructural, holístico holográfico) y empíricos de tipo cualitativo y cuantitativo, es así que, la investigación cualitativa se centró en el significado de la realidad formativa de futuros maestros en torno al proceso enseñanza y aprendizaje, cómo se interpretó más allá de las distintas corrientes pedagógicas y la experiencia misma. Por lo que, a través de la investigación cualitativa se ha formado parte del objeto de estudio para la recogida de datos utilizando guías de observación. Así mismo, la investigación cuantitativa permitió recolectar la información a través de encuestas y entrevistas a los estudiantes, docentes y directivos de las Escuelas Superiores de Formación de Maestros, con la finalidad de tabular, analizar e interpretar en datos porcentuales y estadísticos.

El análisis del objeto de estudio, a partir de la observación participativa, las entrevistas y las encuestas aplicadas (que se mencionó anteriormente), se dio lugar a la recogida de la información, permitiendo de esa manera recolectar datos y categorizar para determinar el impacto del problema y la necesidad de resolverlo, a partir de la fundamentación teórica concretada en la hipótesis que permitió determinar los componentes, relaciones esenciales, estructuras y regularidades en un nuevo modelo teórico educativo que se sustenta en la búsqueda y transformación permanente del profesorado hacia el desarrollo de la educación dialógica y participativa entre sus actores educativos, permitiendo construir y reconstruir conocimientos de manera integral y holística.

RESULTADOS

\section{Realidad formativa de futuros maestros en las Escuelas Superiores, a partir de los resultados de investigación cualitativa}

A través de la investigación cualitativa, que se muestra en la siguiente Tabla 1 centralizador de actividades de visita a las Escuelas Superiores de Formación de Maestros (ESFM), aplicando las guías de observación, tiene como finalidad conocer la realidad formativa de futuros maestros en las instituciones mencionadas a continuación. Considerando que el objetivo de este escenario investigativo, fue determinar la problemática de mayor impacto como resultado.

Tabla 1. Actividades de visita a las ESFM y resultados de observación.

\begin{tabular}{|c|c|c|}
\hline ESFM & ACTIVIDADES DE VISITA & RESULTADO \\
\hline “SIMÓN BOLÍVAR” & $\begin{array}{l}\text { - Observación de clase } \\
\text { - Observación general a la ESFM } \\
\text { - Observación del aspecto formativo } \\
\text { que desarrollan los docentes en cada } \\
\text { carrera o especialidad de formación }\end{array}$ & $\begin{array}{l}\text { - Ausencia de coordinación y diálogo } \\
\text { durante las actividades de clase } \\
\text { - El proceso formativo que desarrolla } \\
\text { cada docente es aislado y muy } \\
\text { individualista }\end{array}$ \\
\hline $\begin{array}{c}\text { “MANUEL } \\
\text { ASCENCIO } \\
\text { VILLARROEL" }\end{array}$ & $\begin{array}{l}\text { - Observación de actividades de clase } \\
\text { - Observación del comportamiento } \\
\text { del personal docente, directivo y } \\
\text { administrativo, respecto a la } \\
\text { formación de futuros maestros }\end{array}$ & $\begin{array}{l}\text { - Los docentes no toman en cuenta la } \\
\text { pluralidad y diversidad de aprendizaje } \\
\text { de los estudiantes } \\
\text { - Las actividades de clase son } \\
\text { desarrolladas de manera aislada } \\
\text { - No existe diálogo ni intercambio de } \\
\text { experiencias formativas, trabajan de } \\
\text { manera individualista. }\end{array}$ \\
\hline
\end{tabular}


ESFM

“ÁNGEL

MENDOZA

JUSTINIANO"
ACTIVIDADES DE VISITA

- Observación general de la ESFM

- Observación de las actividades de aula que desarrollan los docentes
“FRANZ TAMAYO” - Observación al desempeño de los docentes, estudiantes y directivos dentro la ESFM

- Observación de la relación personal y profesional de los docentes y directivos en la ESFM

$\begin{array}{ll}\text { “SIMÓN BOLÍVAR" } & \text { - Observación de clase } \\ & \text { - Observación general a las } \\ & \text { actividades académicas e } \\ & \text { institucionales de la ESFM } \\ \text { “JUAN MISAEL } & \text { - Observación a las actividades } \\ \text { SARACHO" } & \text { académicas desarrolladas dentro del } \\ & \text { aula } \\ & \text { Observación general a la ESFM }\end{array}$

“ENRIQUE FINOT” - Observación a las actividades de aula

- Observación general a las actividades académicas, respecto a la preparación para las prácticas educativas
- Observación al desempeño de los docentes en aula

- Observación general a la ESFM

\section{RESULTADO}

- No existe diálogo, ni intercambio de experiencias conjuntas entre docentes y con los estudiantes.

- El proceso enseñanza aprendizaje que desarrollan los docentes es aislado.

- No existe consenso, ni diálogo participativo entre docentes, directivos y estudiantes en el proceso formativo de futuros maestros.

- Se identifican conflictos de interacción, ego profesional, individualismo $\mathrm{y}$ trabajo aislado.

- Los docentes trabajan de manera aislada.

- No existe cultura de trabajo en equipo.

- No existe coordinación, ni diálogo en aspectos pedagógicos y didácticos de la formación.

- No contemplan la diversidad educativa de los estudiantes del sistema regular, las experimentaciones pedagógicas $\mathrm{y}$ didácticas desarrolladas en la ESFM.

- Se desconocen los requerimientos educativos que demanda la educación regular.

- Las prácticas educativas de los futuros maestros no respetan la diversidad cultural.

- No se diagnostican los conocimientos previos de los estudiantes.

- No existe interacción de experiencias educativas entre docentes y estudiantes.

- Trabajo académico poco coordinado.

- No se trabaja de manera cooperativa y compartida las actividades pedagógicas y didácticas de aula.

- Los conflictos de interacción entre los actores educativos, limita el trabajo colaborativo en la ESFM. 
“PUERTO RICO” - Observación general a la ESFM

- Observación al trabajo académico de aula

- Observación a los aspectos pedagógicos y didácticos que se desarrollan en aula
- Insuficiente preparación de los futuros maestros, en los aspectos psicopedagógicos y psicosociales.

- No existen acciones educativas de interacción entre ESFM, para mejorar la calidad formativa de futuros maestros.

- No existe diálogo, ni intercambio de experiencias entre los actores educativos.
Por lo tanto, el análisis de los resultados de esta fase de investigación, determinó que en las Escuelas Superiores, la formación de maestros es aislada e individualista a consecuencia de conflictos de interacción entre actores educativos que no permite desarrollar un trabajo colaborativo e interactivo. Entonces el proceso formativo de futuros maestros no es dialógico ni participativo.

\section{Sistematización de resultados investigativos cuantitativos, respecto a la naturaleza formativa de maestros en las Escuelas Superiores}

Realizado el estudio a través de los instrumentos de investigación, se ha llegado a los siguientes resultados cuantitativos, que se presenta en el siguiente gráfico 1.

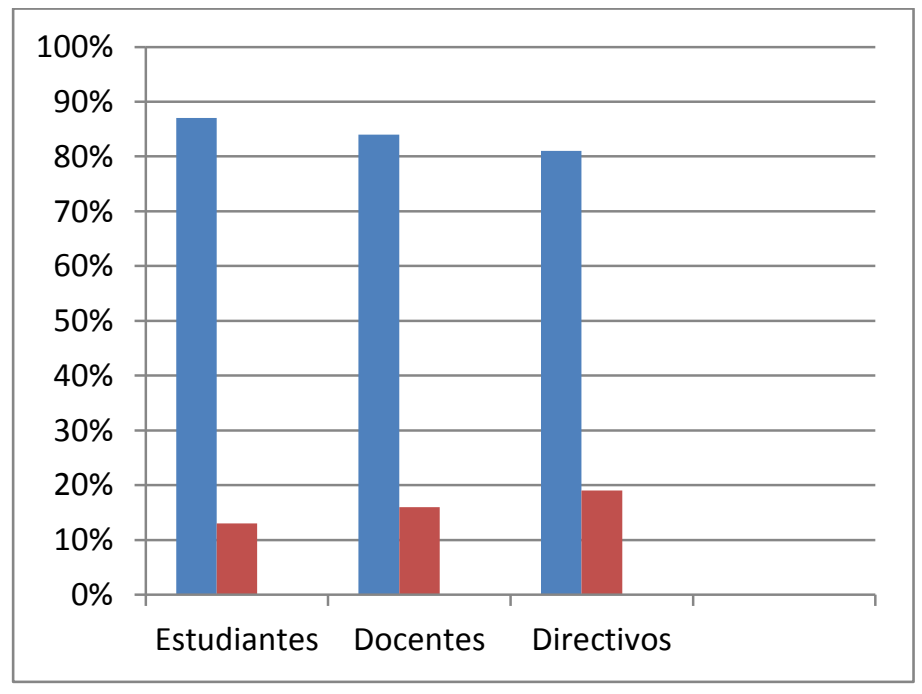

Gráfico 1. Resultados de encuestas y entrevistas a los estudiantes, docentes y directivos

El análisis e interpretación del Gráfico 1 , demuestra que el $87 \%$ de los estudiantes (futuros maestros) expresan: que las actividades pedagógicas y didácticas de aula se desarrollan de manera aislada e individual, no es participativo, no se practica el diálogo ni la coordinación, son poco colaborativos y la interacción docente y estudiante es insuficiente. El 13\% expresa lo contrario. 
Así mismo, el $84 \%$ de los docentes (formadores de maestros): no tienen cultura de trabajo en equipo, las clases desarrollan de manera aislada, no intercambian, ni comparten experiencias educativas con sus colegas $\mathrm{y}$ estudiantes. El 16\% no comparte el criterio expuesto.

Por otra, el 81\% de los directivos: alguna vez dialogan y participan en aspectos formativos, no coordinan, ni comparten experiencias con docentes y estudiantes, ni con otras ESFM, por lo que el 19\% expresa lo contrario.

Por lo tanto, la realidad educativa respecto a la formación de maestros en las Escuelas Superiores, admite que hay insuficiente interacción docente - estudiante y docente docente, lo cual no posibilita intercambiar experiencias educativas en el desarrollo de los contenidos y habilidades en el proceso de enseñanza aprendizaje. En consecuencia, los docentes desarrollan el proceso formativo de manera aislada e individual, lo que no da lugar a coordinar y dialogar acciones educativas.

Los resultados del diagnóstico de tipo cualitativo y cuantitativo, sobre la realidad formativa de los futuros maestros, y teniendo como sustento los instrumentos que fueron aplicados, indican que existe un problema: insuficiencias de interacción dialógica $y$ participativa en el proceso de preparación pedagógica y didáctica de los futuros maestros. Este problema se revela en el sentido de que el proceso educativo/formativo se desarrolla de manera aislada e individual como se ha demostrado, lo cual no permite intercambiar experiencias educativas entre docentes $\mathrm{y}$ estudiantes de manera dialógica y participativa

En fin, en esta realidad educativa, se observan conflictos de interacción en el trabajo de aula de los docentes, que inciden en el desarrollo de las habilidades pedagógicas y didácticas de manera integral en el futuro maestro.

\section{Resultado del análisis y fundamentación de solución}

Conociendo las causas que dificulta desarrollar las habilidades docentes en interacción de experiencias en la formación de maestros, se plantea acciones educativas como solución frente a la necesidad latente.

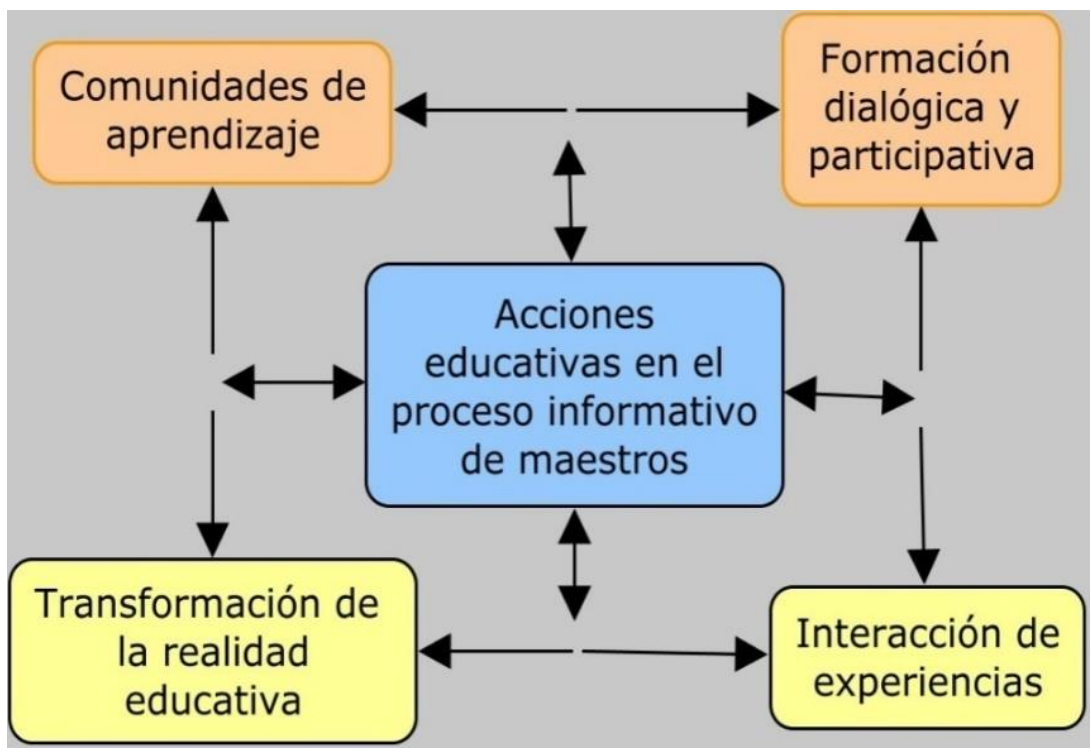

Figura 1. Componentes y relaciones en la dinamización de las acciones educativas. 
En la figura 1 se aprecian los componentes y relaciones principales de las acciones educativas de manera dialéctica e integral, en tal sentido, las comunidades de aprendizaje de cada Escuela Superior dinamizan y operativizan la formación dialógica y participativa de maestros, a través de la interacción de experiencias para transformar la realidad educativa.

Esta interacción de experiencias, consiste en la implementación de acciones educativas en el proceso formativo de maestros desde las comunidades de aprendizaje en las Escuelas Superiores, intercambiando experiencias pedagógicas y didácticas de manera dialógica y participativa, para contribuir a la formación académica e integral del futuro maestro.

Es así que, estas acciones educativas en el proceso formativo de maestros, a través de las comunidades de aprendizaje organizadas en cada ESFM, es factible desarrollar y llevar adelante. Las acciones educativas, dentro de sus características, tiene carácter comunicativo porque a través del intercambio de experiencias educativas, los futuros maestros fortalecen su formación al dialogar y consensuar planteamientos pedagógicos y didácticos del proceso educativo de naturaleza interinstitucional a través de las comunidades de aprendizaje.

Para implementar las acciones educativas en la formación de maestros, se plantean los siguientes principios:

- Las Escuelas Superiores son espacios que deben favorecer la comunicación.

- Las prácticas educativas se desarrollan a partir de tareas específicas.

- Las experiencias y las interacciones profesionales y personales, se desarrollan desde las acciones educativas, en cuya concepción pueden participar representantes de las ESFM.
La comunicación fluida e integradora en estos espacios de formación de maestros, es una herramienta esencial de convivencia y aprendizaje social. Se estimula el respeto por lo diverso, porque inciden en su propia autoestima. Entonces, la Escuela Superior genera en el individuo oportunidades de compartir experiencias formativas, desde tareas específicas, planificadas, que pueden tener como base las experiencias y la interacción entre los profesionales que obran en ella.

La organización de comunidades de aprendizaje de las escuelas, contarán con un equipo promotor que coordine y lidere las actividades, se establecerán tiempos y espacios de formación y reflexión para los diferentes actores que intervendrán, intercambiarán y compartirán experiencias educativas de la misma área de conocimiento y equipos mixtos, planificarán y diseñarán conjuntamente el proceso formativo mediante el desarrollo de habilidades docentes, a través de redes comunicativas e interactivas.

Estas políticas educativas en la formación de maestros, a través de la interacción educativa de las comunidades de aprendizaje, son imprescindibles en la transformación de la realidad formativa de los futuros maestros, por tanto, puede ser factible implementar como propuesta educativa, como nuevo escenario formativo, desde un enfoque dialéctico de (relaciones complementarias y contradictorias) y holístico como (un todo interrelacionado), en la aplicación y desarrollo de las acciones educativas en las ESFM.

En fin las acciones educativas podrán implementarse en el proceso de preparación de los futuros maestros relacionados con su desarrollo y la innovación educativa, el cambio y la transformación educativa en las Escuelas Superiores. Es así que las acciones actuarán como engranajes en la formación integral dialógica y participativa del futuro maestro, frente a la formación individualista y aislada.

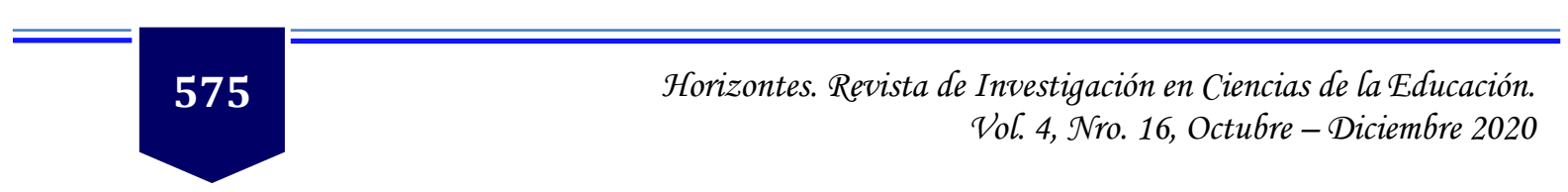


Por los argumentos señalados y desde el conocimiento de la teoría existente acerca del proceso formativo de maestros en las ESFM, sus antecedentes, el diagnóstico del estado actual del objeto, la consideración de las necesidades demostradas mediante el diagnóstico, la determinación de los elementos, propiedades y relaciones esenciales del objeto que se ha caracterizado y la expresión de nuevas propiedades y relaciones que pueden establecerse entre los elementos esenciales del objeto y campo de investigación, las acciones educativas a implementar en el proceso formativo de maestros consisten en:

\section{- SEMINARIOS PERMANENTES de} Especialidad (SPE).

- DINÁMICA DE GRUPOS de tertulia, lectura de teorías educativas.

- ACTIVIDADES DEMOSTRATIVAS AULA TALLER monitoreada por equipos promotores de acciones educativas a nivel nacional y las ESFM.

- INTERCAMBIO DE EXPERIENCIAS respecto a la articulación de Unidades de Formación (UF) y el desarrollo del Proyecto Sociocomunitario Productivo (PSP) en las ESFM.

- DEBATES sobre propuestas educativas basadas en el análisis de experiencias pedagógicas y didácticas planteadas por cada ESFM.

- VISITAS ORGANIZADAS por cada ESFM a lugares históricos, centros culturales $\mathrm{o}$ productivos que sean representativos de la identidad y desarrollo socio - económico cultural de Bolivia.

- CINE - DEBATES: observación de documentales educativos, escogidos colectivamente por cada ESFM, y posterior desarrollo de debates conducidos por comunidades de aprendizaje.
- PANELES: selección colectiva de temáticas a tratar, con panelistas seleccionadas que involucrarán a todos los estudiantes y docentes de cada ESFM, posteriormente se socializará estas experiencias a nivel nacional.

- ENCUENTROS NACIONALES de intercambio de experiencias docentes formadores de maestros.

Todas estas acciones permitirán demostrar experiencias educativas $\mathrm{y}$ formativas a nivel pedagógico y didáctico que planteará cada ESFM para su análisis y validación como aporte a la educación.

\section{DISCUSIÓN}

En el proceso formativo de maestros, inicialmente es importante definir el término proceso, es así que, Álvarez (2016) define proceso como: "...sucesión de los cambios de estados de un objeto en el tiempo". (Álvarez, ob. cit., p. 113), es decir, un proceso implica progresión, desarrollo y transformaciones en etapas determinadas. De forma sintética y con gran carga semántica se concreta el concepto, que al analizarlo, más adelante como proceso formativo, justifica con gran acierto, la precisión expuesta por el autor.

También en ese sentido, Álvarez (2014), escribe:

La instrucción es el proceso y el resultado del dominio de una rama del saber humano, de una profesión; la capacitación, es el proceso y el resultado de formar hombres capaces, inteligentes que hayan desarrollado su pensamiento. La educación es el proceso y el resultado de formar en los hombres su espíritu: sentimientos, convicciones, voluntad, valores, vinculado además a su instrucción y capacitación. (Álvarez, 2014, p. 13). 
La posición del referido autor, es atinada cuando refiere que el proceso es el desarrollo de capacitación, pensamiento, sentimientos y otros aspectos vinculados a la instrucción del hombre, desde la perspectiva de la instrucción y la educación. Por lo tanto, el proceso se define como un conjunto de pasos sistemáticos que aporta al desarrollo y preparación del hombre en todo aspecto.

Por otra parte, lo formativo es algo que contribuye a construir o que sirve para formar, en esa dinámica, en el campo educativo, Álvarez (2014) plantea que "la formación tiene énfasis en la pedagogía que es la ciencia que tiene por objeto el proceso de formación, es decir, la que estudia la formación en general, de la personalidad de los hombres" (Álvarez, ob. cit., p. 19). Esta reflexión permite definir que lo formativo en el aspecto educativo, es la acción de la formación y/o preparación personal y profesional del ser humano en la educación para comprender mejor desde esta perspectiva la manera de pensar de los hombres y la necesidad de prepararlos para la vida. Las ESFM en Bolivia de implicarse en esta idea y alcanzar estadios superiores.

El proceso formativo, entendido como acción o conjunto de acciones o interacciones organizada y planificada entre estudiantes y docentes en el espacio educativo, permite lograr resultados de aprendizaje. Al respecto, Álvarez (2014) menciona sobre "el proceso formativo [...] en la educación, es el proceso social que se desarrolla como sistema para influir en la formación de los rasgos más trascendentes de la personalidad de todos los miembros de la sociedad" (Álvarez, ob. cit., p. 17). Esto significa que el proceso formativo en la educación contribuye a preparar al hombre para la vida, desde la escuela, pasando por un proceso educativo, capacitivo e instructivo, como ya se comenta.
Históricamente y por lo general la formación de maestros siempre ha sido menos atendida en los Estados y sociedades. Sin embargo, el maestro como sujeto activo del aprendizaje necesita estar permanentemente en actualización y capacitación en aspectos pedagógicos $\mathrm{y}$ didácticos, para enseñar $\mathrm{y}$ desarrollar conocimientos de mayor calidad en sus estudiantes.

Una mirada a la formación de maestros, implica responder a las necesidades educativas y formativas de los estudiantes en los centros de capacitación y preparación para ser educadores. En este contexto, Alvarado (2013) expresa: "la educación como proceso, desde las prácticas pedagógicas toma sentido la identidad, se reflexiona sobre la cultura con fundamentos éticos y políticos que son aprendidos $\mathrm{y}$ desarrollados y contribuyen a resolver problemas educativos y sociales" (Alvarado, ob. cit., p. 67). Esta idea permite reflexionar sobre las prácticas pedagógicas que desarrolla un maestro, pero que las desarrolla tomando en cuenta los aspectos culturales, éticos y políticos de un estudiante que pertenece a un determinado contexto social, y que desde esta perspectiva es posible desarrollar conocimiento.

Es determinante que la educación en centros de formación de maestros, sea dinámica y de interrelaciones entre sus actores educativos. En ese sentido; Nieva (2016) expresa:

Estas interrelaciones abarcan a la persona en particular y sus aportes a la construcción de la sociedad, dan lugar a nuevas prácticas, nuevas cosmovisiones, modos específicos de afrontar los retos que le van imponiendo los cambios emergentes de su contexto, su historia y cultura desde la interacción con sus congéneres, dadas sus potencialidades como sujeto particular y social, (Nieva, 2016, p. 45) 
Respecto al planteamiento de Nieva (2016) cuando indica que las interrelaciones personales y colectivas son retos que contribuyen a los cambios y transformaciones de la sociedad, se considera también una manera de innovar y tomar acciones educativas que permita interactuar experiencias pedagógicas para mejorar la calidad de enseñanza y afrontar a los cambios emergentes en la educación. De esa manera, intercambiar posturas y planteamientos teóricos en formación de maestros, permite asumir nuevas prácticas educativas y potenciar la preparación del futuro maestro que estará al servicio educativo.

De esa manera, el maestro tiene su importancia en el proceso formativo de sus estudiantes, en tal sentido, el maestro es la persona que tiene vocación de servicio y desempeña un papel importante ante la sociedad. En el campo educativo, el maestro, es el que guía el aprendizaje del estudiante, entonces, un maestro debe ser consciente del papel que asume en la sociedad, dentro del proceso formativo.

Desde estas reflexiones el ser maestro se define como:

Aquella persona con capacidades $\mathrm{y}$ habilidades educativas, que busca no solo la enseñanza y aprendizaje de sus estudiantes, sino formar en ellos su capacidad de crítica y transformación. Entonces, el ser maestro demanda tiempo y compromiso destinado a la jornada escolar en permanente actualización para responder con eficiencia y eficacia, desde su sensibilidad y profesionalismo ante la sociedad. (Castro, 2007, p. 67).

Lo que define Castro (2007) admite entender que el ser maestro implica alto grado de profesionalidad, puesto que en el aspecto educativo, compromete al educador a estar en permanente actualización y capacitación. Por lo tanto, el ser maestro es tener vocación y compromiso para formar personas en la vida y para la vida.

Por esa razón, la preparación del futuro maestro no es una herramienta más para cumplir con las necesidades estudiantiles, sino es una obligación que debe ser practicada en cualquier institución formadora de maestros. En relación con esta idea, Rodríguez (2017) destaca:

La formación docente es un reto que todo gobierno debe de afrontar ante los nuevos desafíos y desarrollos que se experimentan en el mundo científico tecnológico de la educación. Es por eso que la capacitación de nuevos saberes y la adaptación a la tecnología es una forma estratégica que el docente de estos tiempos debe asumir como herramienta fundamental para absorber los cambios y transformaciones que se experimenta en el área educativa. (Rodríguez, 2017, p. 78).

De acuerdo con lo expresado por Rodríguez (2017), la formación científica, tecnológica, pedagógica y didáctica de maestros, a través de experimentaciones e interacción de experiencias, es compromiso y tarea de las instituciones de Educación Superior formadora de maestros. Sin embargo, es una tarea compleja que exige el dominio de acciones educativas que faciliten el proceso enseñanza aprendizaje y desde una visión más amplia y compleja: el proceso formativo. Por eso, el proceso de aprender a enseñar y de preparar a los hombres para la vida, es también responsabilidad de las ESFM.

La formación y entrenamiento de estudiantes para ser maestros, es muy importante dentro la educación, ya que se presentan retos educativos día a día. En esa 
tarea, el maestro tiene la responsabilidad de formar a los estudiantes en todos los aspectos: instructivos y educativos (desarrollo del pensamiento, lo actitudinal y la formación de sentimientos), y si este no cuenta con los saberes y preparación necesaria, no podrá formar cualidades esenciales en la personalidad de los estudiantes. Es por ello que el maestro debe conocer perfectamente los contenidos de enseñanza para saber lo que se enseña y saberlo enseñar desde la complejidad que caracteriza el proceso formativo.

El maestro de las ESFM tiene la responsabilidad de preparar a los que ocuparán un puesto al frente de las aulas, y esta preparación debe estar acorde con los cambios tecnológicos y acciones didácticas adecuadas que se pueden introducir en los centros de formación de maestros y de forma particular, con el objetivo de satisfacer el encargo social y ser capaces de trasmitir el sistema de conocimientos desde la cultura acumulada por la humanidad.

El proceso de formación de maestros en los diferentes niveles y especialidades, desde la generación de lineamientos educativos con una estructura nacional, departamental, regional y comunitaria autónoma, permite desarrollar la preparación como maestro para desempeñar sus funciones en el sistema de educación. En esa dinámica, la interacción entre escuelas puede generar transformación y capacitación de calidad de futuros educadores. Por lo que; Ruiz (2016) expresa que:

Las Escuelas Superiores Formadoras de Maestros (ESFM) son espacios donde se crea práctica - teoría reflexión y producción de conocimiento pedagógico - didáctico, y ciencia educativa que irradia a todo el contexto social, mejorando la educación y la preparación de los ciudadanos bolivianos en la vida y para la vida. (Ruíz, 2016, p. 32).
Según indica Ruiz (2016), las Escuelas Superiores forman maestros con el conocimiento pedagógico y didáctico acorde con la ciencia educativa relacionada con las tecnologías de la comunicación y las necesidades educativas del contexto que deben ser fortalecidas en el maestro. Se deduce, que las escuelas formadoras de maestros están en la obligación de preparar educadores de calidad, a partir de una serie de acciones educativas que tiende a potenciar su capacitación. Por tanto, el desarrollo de conocimientos en estas instituciones no es único, más bien abre otras opciones y rompe con las estructuras rígidas de generar conocimiento, posibilitando el pluralismo de ideas y posturas, los diálogos entre distintos actores educativos y de distintas instituciones, que puede permitir desarrollar prácticas educativas innovadoras y dinámicas. En este sentido Mardesich (2009) escribe que la:

Formación del docente [...] tiene espíritu innovador y creatividad, investigación continua, análisis crítico, una sólida base científica, un abordaje holístico de los saberes, participación activa en la construcción de los aprendizajes, el papel del guía orientador en los procesos de enseñanza - aprendizaje, actitud abierta y autónoma, educación contextualizada que revalorice su propia cultura, respeto a la diversidad, cooperación, trabajo con otros y la conciencia de necesidad de una formación permanente. (Mardesich, 2009, p. 55).

Tomando partido sobre los aportes de Mardesich (2009), es importante considerar que la formación docente, tiene espíritu creativo e innovador, es así que, el proceso formativo de maestros en la Escuelas 
Superiores, debe caracterizarse por la alta responsabilidad que deben tener todos sus actores educativos, puesto que se demanda desarrollar en el futuro maestro, una preparación sólida que sea capaz de impulsar un conocimiento que revalorice las culturas ancestrales y las universales, desde una perspectiva pedagógica dialéctica, y de manera cooperativa con otros.

El autor comparte las ideas de Ruiz (2016) porque la ciencia educativa se encuentra en todo el contexto social y debe tener un espíritu innovador, de creatividad y de investigación permanente, capaz de involucrar en su accionar a todos los actores educativos de manera holística y dialéctica en su procedimiento educacional y teniendo como plataforma las dos funciones básicas en el proceso formativo: instrucción y educación.

La formación de maestros en las Escuelas Superiores, está a cargo de docentes y directivos, que son profesionales que cuentan con un nivel de formación igual o superior al que están formando. Estas instituciones son de carácter Estatal, y se encuentran bajo la administración del Ministerio de Educación.

La importancia del trabajo metodológico dentro de los colectivos docentes, para que los contenidos en la carrera docente sean trabajados de manera eficiente, es decir, las teorías educativas que se abordan en los centros de formación docente, contribuyan satisfactoriamente a la formación pedagógica y didáctica del futuro maestro y estar al servicio de la educación en la actualidad, demanda preparación de calidad, según las investigaciones de la realidad educativa en las Escuelas Superiores. En esta dirección, Álvarez (2014); expresa:

La Pedagogía [...] consiste en significar sus fundamentos conceptuales y sistema categorial, objeto, dimensiones, cualidades, componentes, leyes y eslabones, que permite concluir que, sobre la base de sus propios métodos científicos, es posible el análisis y la solución de los problemas que con más fuerza se revelan en la escuela contemporánea. (Álvarez, 2014, p. 22).

Las ideas de Álvarez (2014) respecto a la Pedagogía destacan la importancia de la formación pedagógica del futuro maestro, puesto que el significar los fundamentos conceptuales, dimensiones y cualidades como procedimientos metodológicos de la enseñanza aprendizaje, que permita superar los problemas educativos en la escuela contemporánea, y más aún en centros de formación docente, reconoce que se puede responder a las exigencias educativas de calidad, en correspondencia con las características y exigencias del momento que se vive.

Por otra parte, la Pedagogía es considerada como la ciencia que estudia la metodología y las técnicas que se aplican en la práctica educativa. En ese sentido, la Pedagogía también es definida como:

La ciencia que orienta las acciones educativas en base a ciertos pilares como la: práctica, técnicas, principios y métodos que tiene como objetivo desarrollar los procesos de enseñanza y aprendizaje desde los principios psicológicos, socio culturales y lingüísticos de los estudiantes que pertenecen a un contexto social diverso. (Reffino, 2019, p. 33).

Se comparten las ideas de Reffino (2019), por lo que es atinado determinar que la Pedagogía es una ciencia que se ocupa de estudiar los procesos educativos que se desarrollan en la práctica, es decir, las técnicas, principios y métodos que se utilizan en los 
procesos de enseñanza y aprendizaje en cualquier nivel de formación y sobre todo en el proceso formativo de maestros, es imprescindible. En las ESFM, se desarrolla y se experimentan los procedimientos de enseñanza, es por eso su importancia para lograr la educación de calidad en todo sentido.

Por ello, la educación necesita un cambio, y este cambio debe partir de la carrera docente que se desarrolla en las instituciones formadoras de maestros. En esa nueva acepción, las habilidades específicas del profesorado deben responder a la realidad educativa actual, desde sus dificultades y posibilidades, como el desarrollo de la informática y sus consecuencias desde un enfoque dialéctico y holístico, de forma tal, que el egresado sea capaz de integrar saberes, tomar partido y resolver problemas a partir de una nueva visión de la pedagogía compleja y la caracterización científica del Proceso Enseñanza Aprendizaje (PEA) desde la Didáctica.

Se recuerda que la Didáctica según La Torre (1993) es: "una disciplina pedagógica que se ocupa de los procesos de formación en contextos deliberadamente organizados" (La Torre, ob. cit., p. 81). Este planteamiento orienta organizar los procesos formativos en cualquier contexto educativo. Así mismo, según Toro (2014) la Didáctica es: "una disciplina de la enseñanza del conocimiento cuyo objetivo es el entendimiento, mediante unos principios pedagógicos encaminada a una mejor comprensión de las ciencias" (Toro, ob. cit., p. 34). Esta idea es una definición esencial que se da para un proceso educativo, porque, se considera a la didáctica como aquella ciencia que estudia el proceso enseñanza aprendizaje en la formación del individuo desde el desarrollo de las clases en aula.

De otra manera, Álvarez (2014) define la Didáctica como: "la ciencia que estudia el PEA o proceso formativo eficiente", y expresa: " $L a$ didáctica nos permite dirigir científicamente la formación de los hombres de una sociedad" (Álvarez, ob. cit., p. 19). Estos planteamientos e ideas se comparten plenamente por el autor porque se refieren a la preparación del hombre para la vida, lo que significa desarrollar el proceso de formación de manera integral y sobre la base de la preparación eficiente y especializada del maestro, para desarrollar conocimientos y habilidades en los estudiantes en cualquier nivel de formación.

En ese sentido, la formación pedagógica y didáctica de maestros en la Escuela Superior, contribuye a desarrollar las habilidades educativas que todo maestro debe conocer $\mathrm{y}$ llevar a la práctica educativa en beneficio de los estudiantes. Es por eso que en las ESFM los docentes debieran dialogar e intercambiar experiencias educativas que puedan enriquecer la preparación pedagógica y didáctica del futuro maestro.

Por tanto, el trabajo metodológico en la carrera docente, debe ser comprometido y capaz de abordar aspectos educativos/formativos de manera dialógica y participativa entre los actores educativos de la ESFM, teniendo como fundamentos científicos la Pedagogía y la Didáctica. Desde estas categorías conceptuales aludidas y teniendo como premisa el modelo del hombre a formar y lo complejo del proceso de formación de maestros que alude a la primera y segunda ley de la pedagogía, declaradas por Álvarez (2011) se plantea que la preparación integral de los ciudadanos de un país para satisfacer las necesidades sociales es el problema fundamental de la Pedagogía.

$\mathrm{Al}$ referirse al modelo de hombre a formar, Álvarez (2011) escribe en su libro Pedagogía:

Para satisfacer la necesidad, el problema, de la preparación integral de los ciudadanos de una sociedad hace falta educarlos. Es decir, que la respuesta a la 
necesidad de la preparación se alcanza si los ciudadanos están educados, que constituye el objetivo a lograr. (Álvarez, 2011, p. 104)

Se comparte el planteamiento de Álvarez (2011) respecto a la preparación integral de los ciudadanos, donde hace falta educarlos, es decir, insiste en la idea de la educación como resultado a alcanzar, porque este acto puede desarrollarse en la escuela de educación regular o en otros centros de educación superior y en la familia, mediante acciones que todo hombre debe practicar en su cotidianidad para poder formarse y resolver los problemas que tiene que enfrentar. El autor mencionado se refiere a la estructura compleja de la educación y a la necesidad de asumir el proceso formativo en todos los aspectos de la personalidad del estudiante.

En lo relacionado con la segunda ley de la Pedagogía, Álvarez (2011) escribe:

El ciudadano diariamente se ve en la necesidad de apropiarse de un contenido, de una rama del saber para que, utilizándola como herramienta, pueda desarrollar su profesión (la instrucción). Esa es, justamente, la vía que le posibilita, debidamente encauzada, lograr en su formación atributos de la subjetividad de la personalidad, del ser, que le hacen afín a su papel de ciudadano, la educación.(Álvarez, 2011, p. 113).

Reflexión significativa la de Álvarez (2011) cuando expresa la necesidad de apropiarse de un contenido diariamente, es decir, de instruirse, es lo que cada persona debe realizar todos los días, es en ese sentido, que el hombre desarrolla su profesión, desarrolla las cualidades de su personalidad, aprendiendo cada día de sus experiencias vividas, así se fortalecen los atributos para desempeñarse en su vida cotidiana, y esto es una forma de educarse y autoformarse cada día.

Son estas posiciones sumamente adecuadas para trabajar conjuntamente, estableciendo relaciones sólidas, orientadas a la resolución de problemáticas educativas, desde estructuras grupales, de los asuntos que tiene la institución por resolver, sin olvidar que, aunque la institución es un referente global necesario, también la mirada del profesor debe proyectarse sobre el aula y los procesos de formación del hombre, así como los didácticos. El compromiso de los miembros de una institución formadora de maestros debe ser de transformación e innovación educativa permanente.

La formación permanente del profesorado se constituye en un derecho y en una obligación de las administraciones educativas y de las propias escuelas formadoras de maestros. Entonces, estas escuelas deben priorizarla formación de calidad del profesorado, de modo que se orienten a la resolución de los problemas educativos reales de los maestros, desarrollando la reflexión, que ha de facilitar el juicio crítico y el trabajo en equipo.

La educación es un proceso bidireccional que modifica las formas de conducta humana ya que se transmiten nuevos conocimientos, valores, costumbres y formas de actuar. Sin embargo, a partir del problema fundamental de la Pedagogía, existen múltiples problemas que se presentan en la educación y en todos los niveles, una de ellos, es el trabajo aislado del docente que no utiliza acciones educativas colaborativas, participativas y dialógicas en la Educación Superior. Es por eso que el futuro maestro tiene escasa preparación respecto a su formación pedagógica y didáctica.

Entonces, la formación eficiente del futuro maestro depende también de su desarrollo formativo en las Escuelas Superiores, escenarios donde se prepara pedagógica y didácticamente, 
analizando y reflexionando sobre procedimientos educativos, metodologías, perspectivas y planteamientos teóricos del quehacer educativo y muchos otros que permitan alcanzar preparación de calidad del maestro.

Por lo que el proceso enseñanza aprendizaje en formación de maestros, debe ser científico y técnico, donde se tome posturas educativas actualizadas y dinámicas. De esa manera; se declara que:

El hecho educativo responde a una realidad social que nace espontáneamente de las necesidades educativas más sentidas, que requieren atención desde la transformación e innovación pedagógica en los centros de formación docente, para ofrecer a los profesores y alumnos las mejores condiciones para su formación en la educación regular. (Pérez, 2002, p. 56).

En concordancia con el criterio de Pérez (2002) y las ideas de Álvarez (2011), el trabajo pedagógico y didáctico en las instituciones formadoras de maestros, debe capacitar y preparar al futuro maestro para responder a las necesidades educativas del contexto social. En esa dirección existe la necesidad de innovar y transformar el proceso formativo del profesorado, implementando sistemas de acciones educativas plurales, dialógicas $\mathrm{y}$ participativas que permita interaccionar desde las comunidades de aprendizaje de una institución con otra, para mejorar la calidad formativa del futuro maestro, capaz de responder satisfactoriamente a las necesidades educativas, sociales $\mathrm{y}$, en resumen, a la preparación del hombre para la vida.

El proceso formativo de maestros requiere tratamiento para mejorar la preparación académica. En esta dinámica, la interacción es una oportunidad y alternativa educativa que puede favorecer el proceso formativo de maestros, desde las dimensiones: instructivas, desarrolladores y educativas. Para Guevara (2011):

La interacción en el proceso formativo de maestros, es primordial en el proceso enseñanza - aprendizaje a nivel de desarrollo pedagógico y didáctico, entonces la interacción es una experiencia educativa en la que el estudiante aprende con respecto a los contenidos, en estrecha interrelación entre: estudiante - docente, estudiante - estudiante, docente - docente, directivo - docente, dependiendo la forma en que se proyecte la práctica educativa mediante el canal comunicativo. (Guevara, 2011, p. 10).

De acuerdo con los criterios de Guevara (2011), la interacción de experiencias educativas en un proceso de enseñanza aprendizaje a nivel de formación de maestros, es de mucho beneficio para mejorar su calidad formativa, puesto que la interrelación de experiencias en las ESFM y entre estas instituciones, a través de redes comunicativas y las comunidades de aprendizaje, permite dar solución a las problemáticas educativas debido a insuficiencias en cuanto al diálogo y a la educación participativa en los centros de formación docente, entre otras situaciones que puedan existir.

La interacción fomenta la unidad de los actores educativos en el proceso formativo, desde las comunidades de aprendizaje de cada Escuela Superior. De esa manera, los centros de formación de maestros se implican en la revolución del aprendizaje, se convierten en animadores y líderes, y por ello, tienen la misión y la responsabilidad de un trabajo orientado hacia la comunidad y una dinámica para el desarrollo de estas, formando redes de 
sujetos comprometidos en el desarrollo del conocimiento colectivo que genera intercambio de experiencias profesionales y la posible solución de problemas.

Se destaca cómo en las comunidades de aprendizaje parten de un principio regulador que es el aprendizaje dialógico. Según Coll (2006): "la concepción comunicativa del aprendizaje depende de las interacciones y el diálogo consensuado entre personas, de la construcción conjunta de significados, un modo de desarrollar el diálogo reflexivo a través de grupos interactivos [...] por cooperación y colaboración" (Coll, ob. cit., p. 88). Esta mirada de Coll, ob. cit., establece que los grupos se amplíen y participen entre todos los actores educativos en un determinado nivel, por lo que el objetivo fundamental de la participación en una comunidad de aprendizaje, es movilizar a toda la comunidad educativa: profesorado, directivos, estudiantes y agentes educativos, ya que una comunidad de aprendizaje, no sólo orienta hacia el éxito académico, sino también el éxito social y el desarrollo personal. En consecuencia es oportuno referirse a la comunicación y su importancia en el proceso de enseñanza aprendizaje.

Es así que, para Cruz (2013):

La comunicación en el aula debe ser una relación terapéutica entre docente y estudiante, un auténtico encuentro entre seres humanos que aspiran a la optimización de los aprendizajes. Si la relación es adecuada, el trabajo del docente es realizado con mayor calidad y las situaciones son desarrolladas favorablemente para él mismo y para los alumnos. De esta manera, una positiva relación entre docentes y estudiantes, y estudiantes entre sí, a través de la comunicación [...] que promueve la calidad en el proceso enseñanza aprendizaje. (Cruz, 2013, p. 5)
Lo que expresa Cruz (2013) respecto a la comunicación y al referirse a la relación docente - estudiante, como terapia, se considera que genera un ambiente de confianza y de trabajo en el aula. El hecho de comunicarse entre actores educativos de manera adecuada, puede permitir generar aprendizajes óptimos y de calidad.

Por otro lado, según González (1995) expresa que "la base de la educación es precisamente la comunicación. A través de la comunicación se brinda la enseñanza y a su vez se ejerce una influencia educativa sobre el escolar en un medio participativo" (González, ob. cit., p. 133). Estos planteamientos coinciden con la importancia que Álvarez (2011) otorga a la teoría de la comunicación. El proceso comunicacional es un elemento esencial en el proceso educativo $\mathrm{y} / \mathrm{o}$ formativo.

Teniendo como soporte lo dialógico, lo comunicacional dentro del proceso formativo, es pertinente abordar ideas sobre la participación de los equipos interactivos en una comunidad de aprendizaje ya que, contribuye al cambio y el desarrollo de la educación en cualquier nivel de formación.

Los equipos interactivos desarrollan un aprendizaje dialógico en las comunidades de aprendizaje de las ESFM. En ese sentido, el aprendizaje se construye socialmente desde las interacciones entre personas, a partir de las experiencias profesionales relacionadas con la Pedagogía y la Didáctica que debe manejar un maestro con sus estudiantes. Por ejemplo, en los estudiantes, según Carbonell (2017) afirma que "la construcción de significados se puede desarrollar desde las interacciones que resultan de un diálogo igualitario entre estudiantes, lo mismo ocurre en el profesorado, los familiares, amistades y otras personas" (Carbonell, ob. cit., p. 67). Tal como expresa el autor, en los grupos interactivos se estimula el cambio de roles, el alumnado puede enseñar y en otro momento 
aprender de sus compañeros sobre la base del diálogo igualitario. En los grupos interactivos el alumnado aprende a ayudarse, a compartir esfuerzos, a explicarse las cosas mutuamente de la manera más efectiva, a animarse, a discutir y a ser solidarios, con lo cual se está contribuyendo al desarrollo de la personalidad del estudiante. Además, se garantiza el éxito en el aprendizaje para todos, incluido aquel estudiante que presenta mayores dificultades para aprender.

Por lo tanto, los grupos interactivos (interacción entre personas) como base de las comunidades de aprendizaje dialógico, es parte de la idea de que en todas las personas se pueden dar procesos de cambio. A través del diálogo y en interacción con las demás es cuando de verdad se logra crear canales para superar situaciones de desigualdad y/o de exclusión. Si se enriquecen las intervenciones en el aula con otros profesionales de campos diferentes y otros agentes sociales y culturales de la comunidad, se estará haciendo al estudiantado inteligente, flexible y competente, elevando al máximo sus expectativas de aprendizaje, y estas ideas de sistema de acciones educativas en formación de maestros pueden contribuir a solucionar el problema declarado y lograr la solución. Al respecto, Álvarez (2011) expresa “...la participación activa de profesores y estudiantes que interactúan en el desarrollo de la clase para construir, crear, facilitar, liberar, preguntar, criticar y reflexionar en torno a la comprensión de las estructuras profundas del conocimiento" (Álvarez, ob. cit., p. 27). Se comparte la idea del autor, ya que en lo expresado se infiere la flexibilidad, lo opcional, el acto de intervenir e implicarse y el valor del diálogo.

La participación es un recurso esencial de una comunidad de aprendizaje, contribuye al cambio y oportunidad de desarrollo, desde diversas aristas y en correspondencia con las demandas sociales. De esa manera:

El efecto general en una comunidad
formativa, es hacer del cambio una
oportunidad y se destaca la
importancia de la asociación,
innovación y un enfoque de abajo
hacia arriba. Entonces, el ímpetu y
la planificación vendrán de la
energía de los líderes. Y la cuestión
importante es definir en cada
equipo interactivo el qué, el cómo,
el cuándo y el dónde se va a
desarrollar, y hacer los esfuerzos
necesarios para incrementar la
participación de los distintos
grupos de profesionales. (García,
2005, p. 204).

Sobre la base de la posición de García (2005) uno de los primeros elementos y tareas de sus miembros es la participación de los integrantes de la comunidad formativa en la revisión de los programas y actividades que orientan el funcionamiento del equipo interactivo, donde se pueden integrar diversas funciones como: construir la comunidad, facilitar el desarrollo profesional, el desarrollo personal, y el reconocimiento y valoración de los colegas.

Las proyecciones de las comunidades de aprendizaje, involucran a las personas, instituciones formadoras de maestros y otras instituciones educativas. Esta interacción o interrelación genera participación y diálogo que persiguen objetivos comunes en el proceso enseñanza aprendizaje. Es así que:

Las Comunidades de Aprendizaje tienen como objetivo, la creación de espacios que permitan el ejercicio de una ciudadanía responsable y sostenible. Son proyectos que pretenden, sobre todo, crear un marco de participación de todas las 
personas e instituciones sociales en la tarea de formación de sus ciudadanos, ya sea en el campo educativo o en otro rubro. (Tam, 2004, p. 165)

Compartiendo el planteamiento de Tam (2004) la creación de espacios de comunidades de aprendizaje como proyectos de interacción de experiencias de carácter educativo y social, puede contribuir al desarrollo de colectividades en el contexto del diálogo y participación entre sus miembros. En ese sentido, las comunidades formativas de aprendizaje como proyectos de participación social y/o educativa, requieren un cambio profundo de mentalidad, no puede efectuarse sin tener en cuenta la realidad.

La transformación de los centros educativos y Escuelas Superiores de Formación de Maestros en comunidades de aprendizaje, se basa en el cambio de las ideas que emergen de la sociedad actual y en abrirlas puertas de la escuela a la comunidad para entre todos plantear los problemas que interesan. Las comunidades de aprendizaje según Coll (2004) surgen como:

Reactivo social ante la incapacidad de la educación formal para responder a las nuevas y emergentes necesidades de las generaciones actuales y venideras, siendo necesario compartir lo bueno y lo malo del contexto social y educativo, basado en los principios de: crear el conocimiento adecuado, encontrar socios y estar comprometidos en el desarrollo de los proyectos educativos y sociales. (Coll, 2004, p.124).

De acuerdo con las ideas de Coll (2004), es importante reiterar, que ni personalmente ni en el ámbito de la empresa educativa o cualquier corporativo social e institucional, se puede esperar que sea el otro, el gobierno u otras compañías, quien dé los primeros pasos para transformar las instituciones, sino que se debe actuar rápida $\mathrm{y}$ eficazmente frente a sus necesidades en la manera de dirigir y llegar a los objetivos trazados, es decir, las comunidades de aprendizaje son escenarios de interacción educativa como reactivos en la ESFM, con la finalidad de generar conocimiento, para que contribuya a la formación de maestros en el campo de la Pedagogía y la Didáctica.

Es así que los proyectos educativos de interacción del conocimiento, son sistemas de acciones educativas que fortalecen el proceso formativo de futuros maestros que estarán al servicio de la educación.

El trabajo colaborativo en el desarrollo de la educación en cualquier nivel de formación, es generado al interior de las comunidades de aprendizaje, esto es parte de un proceso enseñanza aprendizaje dialógico y participativo, que enriquece el proceso formativo.

Por lo que, Lanni expresa:

La convivencia escolar, desde este ángulo, alude, fundamentalmente, a uno de los temas básicos de la pedagogía y el aprendizaje, es decir, el proceso por el cual un sujeto adquiere o desarrolla una nueva conciencia y conocimiento, que le proporcionan nuevos significados. Para que el aprendizaje sea posible, los intercambios de todos los actores de la institución (estudiantes, docentes y padres de familia) que comparten la actividad en la escuela y que conforman esa red de vínculos interpersonales que genera el denominado convivencia. (Lanni, 2003, p. 145).

De acuerdo con Lanni (2003), es necesario generar vínculos interpersonales de carácter colaborativo, que permitan practicar una 
convivencia que posibilite intercambiar e interactuar experiencias personales $y$ profesionales para coadyuvar en el aprendizaje significativo y productivo de los estudiantes de cualquier nivel de formación. En el caso de formación de maestros, la interacción de experiencias pedagógicas y didácticas a través de acciones educativas dialógicas y participativas entre comunidades de aprendizaje de cada ESFM, puede contribuir a la preparación de calidad del futuro maestro.

La colaboración y cooperación en el desarrollo de la clase, entre estudiantes, incrementa el aprendizaje para todos. De hecho, ya es una realidad en la educación la tendencia colaborativa. En esa línea, la colaboración y cooperación es un recurso importante en toda institución u organización, ya que en ella las personas se interrelacionan a través de ideas y propuestas de aprendizaje, que tienden a mejorar la educación en cualquier nivel de formación, sea en el ámbito de educación regular o educación superior.

Las características de una convivencia colaborativa en el proceso formativo de maestros, induce a la práctica de una cooperación mutua y recíproca entre los actores educativos desde sus experiencias, a fin de contribuir al desarrollo de las habilidades docentes del futuro maestro.

Por otro lado, la descentralización de responsabilidades en el tema de la educación, está enfatizando lo que es la educación participativa en los últimos tiempos, es así que, una educación participativa y descentralizada, puede definirse como gestión educativa donde la toma de decisiones no está centralizada en un solo nivel, sino que se desplaza a otras instancias que no suelen ser tomadas en cuenta. Esto facilita dar respuestas oportunas a las necesidades específicas en el ámbito educativo, para poder llevar adelante la educación participativa, como lo que refiere Choque (2015):

La educación participativa, comunitaria, democrática y de consensos, por ser un proceso de transformación humana de convivencia con pertinencia, donde se aprende y se práctica la cultura que vive la sociedad o la comunidad educativa, de esta manera, se recrea un proceso de apropiación social y cultural, sin perder su condición de actor social, es así que, las y los estudiantes aprenden todas las prácticas comunitarias, de forma práctica, participativa, democrática y en consenso, en una relación de convivencia social consciente $y$ comunitaria, que es plasmada en la práctica educativa. (Choque, 2015, p. 22).

Desde la posición de Choque (2015), es muy importante tomar en cuenta el contexto en un sistema de acciones educativas participativas, que descentraliza responsabilidades desde un nivel central de educación, hasta los centros educativos que adoptan estrategias metodológicas para mejorar el proceso enseñanza aprendizaje. Se considera que la educación participativa, comunitaria, democrática y de consensos, puede contribuir a un proceso de transformación educativa, que involucre a todos los actores educativos en la tarea formativa de nuevos ciudadanos, a partir de la práctica de una educación participativa.

Según Oramas (2002), determina: "la metodología participativa y su aplicación en el proceso educativo brinda las posibilidades de trabajar en grupo y lograr los objetivos trazados" (Oramas, ob. cit., p. 55). Esta idea orienta a que el proceso educativo/formativo en las Escuelas Superiores sea inclusivo y plural, que permita tomar acciones educativas 
dialógicas y participativas en consenso, para fortalecer la preparación pedagógica y didáctica del futuro maestro.

La escuela como precursor de la teoría y práctica pedagógica, transforma el enfoque tradicional de aprendizaje centrado en el maestro, a un aprendizaje activo centrado en el estudiante, a partir de un proceso de construcción del conocimiento colaborativo y cooperativo, que integra en los procesos de aprendizaje, la relación maestro - estudiante y maestro - comunidad educativa.

Esta realidad educativa, permite desempeñar un aprendizaje activo, cooperativo y participativo centrado en el estudiante, por tanto:

La importancia de poner al estudiante en el centro del aprendizaje, resulta innovador, ya que fomenta el desarrollo de una educación integral que forma para la vida y promueve el desarrollo de las destrezas (...) como aprender a aprender, crear, emprender, tomar iniciativa, pensar de forma crítica, liderar el proceso y trabajar en equipo. Todo esto tiene un impacto en los procesos de aprendizaje cognitivo, socio afectivos, y en los valores y actitudes democráticas y de convivencia pacífica (...) a través de pasantías y formación docente. (Londoño, 2017, p. 45).

El planteamiento de Londoño (2017) cuando manifiesta que el proceso de aprendizaje debe ser centrado en el estudiante, desde sus destrezas, potencialidades y habilidades, compartiendo un trabajo pedagógico que involucra a toda la comunidad, es un reto de las instituciones educativas. En el caso de formación de maestros el proceso de aprendizaje está centrado en la preparación pedagógica y didáctica del futuro maestro en las Escuelas Superiores, así mismo, en las Unidades
Educativas del sistema regular donde realizan las prácticas educativas comunitarias. Por ello, el planteamiento de una propuesta educativa para las ESFM, que consiste en acciones educativas desde las comunidades de aprendizaje, para la formación de maestros de manera dialógica y participativa, es considerado una oportunidad para mejorar la formación de maestros.

\section{CONCLUSIONES}

La investigación enfatizó aportes de relevancia en la profundización del problema de estudio, por lo que se tiene las siguientes conclusiones:

Se determina que, en la formación de maestros, en instituciones de Educación Superior, se desarrolla fundamentalmente lo que es la labor del docente para que los estudiantes del sistema de educación regular reciban una educación de calidad. Es así que, las Escuelas Superiores se caracterizan por la alta responsabilidad que tienen con sus actores educativos, puesto que se demanda desarrollar en el futuro maestro, una preparación sólida que sea capaz de impulsar un conocimiento que revalorice las culturas ancestrales y las universales, desde un proceso formativo dialógico y participativo.

Entonces se establece que las comunidades de aprendizaje consisten en la interacción y convivencia educativa donde se comparten experiencias $\mathrm{y}$ procesos pedagógicos $\mathrm{y}$ didácticos para desarrollar una práctica educativa de calidad, por lo que constituyen una alternativa oportuna para tributar a la solución del problema.

Así mismo se considera que, las acciones educativas en las Escuelas Superiores es una herramienta integradora muy importante para las ESFM, ya que, a través de ellas, se comparten experiencias y actividades educativas interrelacionadas que se planifican, organizan, 
ejecutan y se controlan para alcanzar un objetivo determinado, que es la de formar maestros con alto grado de preparación académica en el aspecto pedagógico y didáctico.

\section{REFERENCIAS}

Álvarez de Zayas, C.M. (2016). "Epistemología del Caos" Kipus - Cochabamba - Bolivia

Álvarez de Zayas, C.M. (2014). "Didáctica elemental" Kipus - Cochabamba - Bolivia

Álvarez de Zayas, C.M. (2011). "Pedagogía, un modelo de formación del hombre" Kipus Cochabamba - Bolivia

Alvarado, V. (2013). "Práctica pedagógica y gestión de aula, aspectos fundamentales en el que hacer docente" UNIMAR - Cuba

Castro, J. (2007). "Maestro: Condición social y profesión docente" Universidad Pedagógica Nacional - Colombia

Choque, M.T. (2015). "Educación comunitaria, democrática, participativa y de consensos" La patria, Bolivia

Coll, S. (2004). "Los proyectos educativos y sociales" Morata - Madrid

Coll, S. (2006). "Nuevos escenarios en la gestión educativa" Grau - España

Cruz, Y. (2013). "La comunicación: incidencia en la formación del profesional de la educación" Centro de información y gestión tecnológica de Santiago de Cuba - Cuba

Carbonell, S. (2017). "Participación de familias y diálogo igualitario" Revista el Horizonte Madrid

García, V. (2005). "Equipos interactivos en la comunidad formativa" UAH - Madrid

Guevara, I. A. (2011). "La integración en el aprendizaje" Universidad Veracruzana México

González, F. (1995). “Comunicación, personalidad y desarrollo" Ciencia - Holguín - Revista - Cuba

La Torre, A. (1993). "La didáctica en la enseñanza - aprendizaje como disciplina" Ed. Grau - España

Lanni, N.D. (2003). "La convivencia escolar, una tarea necesaria, posible y compleja" OEI, España

Londoño, C. (2017). “Cambios importantes que están transformando la educación en el mundo" Arauco, Chile

Mardesich, M.L. (2009). "Las Escuelas Superiores en Bolivia y la formación docente" UMSS - Cochabamba - Bolivia

Nieva, J.A. (2016). "Una nueva mirada sobre la formación docente" Ministerio de Educación Superior - Cuba

Oramas, A. (2002). "Los métodos participativos en pedagogía" Uni - rioja, España

Pérez, M. (2002). "El docente desde la perspectiva del desarrollo curricular, organizativo y profesional" Universidad de Jaén - España

Rodríguez, H. (2017). "Importancia de la formación docente" Universidad Autónoma - Estado de Hidalgo

Ruíz, A. (2016). "Formación de maestros y maestras del sistema educativo plurinacional de Bolivia" Instituto Internacional de Integración - La Paz Bolivia

Reffino, M.E. (2019). "La pedagogía” Academia pedagógica - Argentina

Toro, J. E. (2014). "La enseñanza y la didáctica" Paidós - Barcelona - España

Tam, S. (2004). "Participación de instituciones en las comunidades formativas de aprendizaje" Paidós - Barcelona 